\title{
AphidCV 2.0: uma nova abordagem de classificação, contagem e mensuração de afídeos
}

\author{
João Pedro Mazuco Rodriguez \\ University of Passo Fundo \\ Passo Fundo, RS, Brazil \\ Email: 147187@upf.br
}

\author{
Rafael Rieder \\ University of Passo Fundo \\ Passo Fundo, RS, Brazil \\ Email: rieder@upf.br
}

\begin{abstract}
Aphids are a pest of vegetable crops. Counting and classifying aphids helps to assess and predict damage to crops. Traditionally, this process is done manually by an entomologist. With this in mind, this work presents AphidCV 2.0, a software for automatic counting and classification of plate images with these insects. This study shows a new version in Python language, supporting two species, implements a new neural network and improves the performance and accuracy of the first version. Results show a 10-fold reduction in the time to analyze Petri dish images, and a similar accuracy compared to the old neural network. The proposed method is an important step for agricultural research, automating a process subject to fatigue and dramatically increasing the number of samples analyzed in the same time period.
\end{abstract}

Resumo-Afídeos são pestes de culturas vegetais. Contar e classificar afídeos ajudam a avaliar e predizer danos de plantações. Tradicionalmente, esse processo é feito manualmente por um entomólogo. Com isso em mente, este trabalho apresenta o AphidCV 2.0, um software para contagem e classificação automática de imagens de placas com esses insetos. Esse estudo apresenta uma nova versão, em linguagem Python, com suporte a duas espécies, implementa uma nova rede neural, e melhora o desempenho e acurácia da primeira versão. Os resultados mostram uma redução de 10 vezes no tempo de análise de imagens de placas de Petri, e acurácia similar em comparação a rede neural antiga. $O$ método proposto é um importante passo para a pesquisa na agricultura, automatizando um processo sujeito a fadiga e aumentando vertiginosamente o número de amostras de análise em um mesmo período de tempo.

\section{INTRODUÇÃO}

Afídeos são pragas que podem afetar fortemente a produção agrária. Além de fazer a sucção da seiva da planta, os afídeos também transmitem o vírus Barley/Cereal [1]. Em plantações de cereais, diversas espécies podem parasitá-las, como $\mathrm{Si}$ tobion avenae, Rhopalosiphum padi, Schizaphis graminum, Metopolophium dirhodum e outras [1].

Dentre os cereais, está o trigo, o segundo cereal mais produzido no mundo e que possui um alto impacto econômico. De acordo com Dedryver $e t$ al., os afídeos são responsáveis por uma perda média de 700 mil toneladas de trigo na Europa [2]. Devido a sua proliferação extremamente rápida, estima-se que em poucos dias, os pulgões podem realizar uma perda de 7,9 a $34,2 \%$ de uma plantação [3].

O controle e monitoramento dessas pragas em culturas é crucial para diminuir impactos nas plantações. Para tanto, é necessário um acompanhamento da densidade populacional, preferencialmente em períodos semanais [4]. Portanto, a contagem, classificação e mensuração dos afídeos é uma tarefa crítica para estimar fatores de riscos em plantações [5].

Tradicionalmente, o monitoramento é realizado por coleta de amostras em campo, com um entomólogo realizando a contagem por microscópio. Além disso, afídeos costumam ser separados em três diferentes estágios de desenvolvimento: ninfas, ápteros (adultos sem asas) e alados (adultos com asas) [6]. A classificação desses estágios também é crucial para o monitoramento da praga, visto que auxilia na visualização do avanço da população parasita na plantação.

Entretanto, este tipo de metodologia manual possui uma alta taxa de erro, visto que seres humanos são suscetíveis a fenômenos fisiológicos e psicológicos, como fadiga, ilusões visuais e tédio [7]. Em amostras densas, entomólogos também adotam o uso de estimativa a partir da contagem e classificação parcial da amostra, aumentando ainda mais a taxa de erro.

Nesse contexto, foi desenvolvido um software para contagem e classificação automática de afídeos do trigo, o AphidCV, com a intenção de auxiliar o monitoramento com maior precisão e tempo menor de análise [8]. Em sua primeira versão, atingiu-se uma boa taxa de acerto na contagem, classificação de estágios e mensuração de uma única espécie, Rhopalosiphum padi, reduzindo em até 4 vezes o tempo de análise de uma placa em comparação com a contagem manual.

O programa foi escrito na linguagem Java, utilizando um modelo de rede neural artificial pronto, o InceptionV3. A escolha da linguagem se deu pela facilidade principalmente na criação de interfaces. Entretanto, observou-se um gargalo de tempo na tecnologia utilizada, apesar da alta taxa de acerto, e uma possível perspectiva de melhora na taxa de classificação.

$\mathrm{O}$ objetivo desse trabalho foi melhorar os pontos observados, reescrevendo o software na linguagem Python, mais utilizada para funções com imagens e em técnicas de deep learning do que a linguagem Java, além de possuir um maior suporte da biblioteca Tensorflow [9]. Também criou-se um modelo de rede neural próprio, com a intenção de diminuir o tempo de classificação e aumentar a acurácia, e deu-se suporte a mais uma espécie (Schizaphis graminum).

\section{MATERIAIS}

Esse trabalho teve o suporte da Embrapa Trigo (https://www.embrapa.br/trigo), uma das unidades da 
Empresa Brasileira de Pesquisa Agropecuária (Embrapa). Por intermédio deste apoio, foi possível adquirir as imagens com afídeos, juntamente com a classificação respectiva por entomólogos experientes, para o treinamento das redes neurais de forma supervisionada.

Para a implementação da nova versão do software, nomeado AphidCV 2.0, utilizou-se a linguagem Python 3. Ao realizar essa alteração, esperava-se uma diminuição brusca no tempo de processamento de cada imagem, por se tratar de uma linguagem mais utilizada e com maior suporte para redes neurais e tratamento de matrizes do que o Java [9].

Além disso, foram utilizadas as bibliotecas PySide 2 (https://pypi.org/project/PySide2), Keras 2 (https://keras.io) e OpenCV 3.0 (https://www.opencv.org), para o desenvolvimento da interface gráfica, redes neurais convolucionais e algoritmos de processamento de imagens, respectivamente. No backend do Keras utilizou-se o Tensorflow 2 (https://www.tensorflow.org), por ser uma biblioteca bem consolidada de aprendizado profundo.

\section{MÉTOdO}

1) Aquisição: Para a obtenção dos dados necessários para o treinamento das redes neurais, seguiu-se a mesma metodologia de aquisição de dados do primeiro estudo [8] . O método consiste basicamente no escaneamento de placas de Petri contendo os insetos da espécie desejada e um software auxiliar, também desenvolvido pelos autores, o CropAphid, que recorta os objetos que possivelmente são insetos. Após os dois processos, um entomólogo classifica cada recorte de um possível inseto e adiciona nas bases de treino.

O CropAphid também foi reescrito em Python. O processo de recorte utilizado para aquisição de dados é o mesmo utilizado para a análise automática da placa. A imagem escaneada consiste em uma placa, juntamente com uma caixa que bloqueia iluminações externas e adiciona alguns círculos de referências para realizar a conversão de pixel para milímetros.

2) Processamento: Inicialmente, o software recebe a imagem digitalizada e realiza um pré-processamento para detectar apenas a placa, ignorando as bordas da imagem, aplicando a transformada de Hough circular para detectar apenas a área circular que contém os insetos.

Após a detecção da placa, traça-se um limiar (k) para excluir objetos que possivelmente não sejam insetos, como exoesqueletos, asas e sujeiras. Dessa forma, apenas os objetos mais escuros na varredura são mantidos. O limiar é uma variável customizada na interface do software (inicialmente, $\mathrm{k}=115$ para $R$. padi e $\mathrm{k}=132$ para $S$. graminum), já que um dos propósitos é abranger diversas espécies que possuem colorações diferentes. A Figura 1 mostra uma comparação.

Por fim, é feito a varredura com os possíveis objetos de interesse, executando-se função que detecta todos os objetos da placa. Para excluir contornos indesejados, são traçados dimensões máximas e mínimas (em milímetros), com a intenção de detectar apenas insetos, excluindo objetos muito grandes como folhas ou a própria borda da placa. Novamente, os
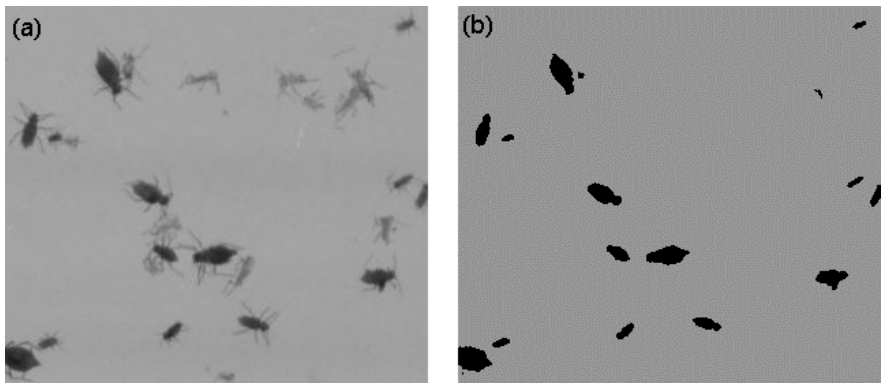

Figura 1. Resultado do thresholding, antes e depois do processamento.

Tabela I

NÚMERO DE IMAGENS UTILIZADAS PARA TREINOS DE CADA CLASSE DAS DUAS ESPÉCIES.

\begin{tabular}{|c||c||c|}
\hline & $R$. padi & S. graminum \\
\hline Ninfas & 7138 & 1062 \\
\hline Ápteros & 6910 & 1190 \\
\hline Alados & 7512 & 1050 \\
\hline Falsos & 7538 & 1032 \\
\hline Total & 29098 & 4334 \\
\hline
\end{tabular}

valores mínimos e máximos são variáveis customizadas na interface, conforme literatura da espécie em análise.

3) Classificação: Após a detecção de objetos que possivelmente são insetos, é feito o uso de inteligência artificial para detectar possíveis falsos positivos que ainda passaram pelos critérios arbitrários anteriores e classificar a fase de desenvolvimento da espécie nas respectivas classes: Ninfas, Ápteros e Alados. As ninfas caracterizam-se por estarem no estágio inicial de desenvolvimento, possuem uma morfologia menos arredondada. Os ápteros são os adultos sem asas, um pouco maiores e mais arredondados e os alados possuem asas.

Para o treinamento supervisionado, treinou-se uma rede neural convolucional própria com quatro classes possíveis: Ninfa, Áptero, Alado e Falsos positivos. Para o retreinamento do modelo para a espécie Rhopalosiphum padi, fora reutilizado a base de treino. $\mathrm{O}$ retreinamento se deu na tentativa de melhorar a acurácia do primeiro trabalho, além disso, houve um aumento de dados dessa espécie, exigindo retreinamento. No total, foram quase 30 mil imagens para treino, com cerca de 7 mil para cada classe. Para a validação, foram utilizadas exatas mil imagens de cada classe. No total, mais de $30 \mathrm{mil}$ imagens de insetos foram analisadas pelo algoritmos para gerar o modelo da rede neural. $\mathrm{O}$ treinamento da espécie Schizaphis graminum contou com uma base menor, com exatos 250 imagens de cada classe para validação. Os valores exatos da base de treino estão descritos na Tabela I.

Notou-se que no estudo anterior haviam algumas possíveis distorções causadas pelo não balanceamento do dataset: havia um número maior de ninfas do que alados, tanto para treino quanto para validação. Isso acabou não refletindo tanto na acurácia durante a realização dos testes, pois as placas usadas nos testes continham mais ninfas, devido principalmente à época de coleta. Entretanto, notou-se que esse fato poderia 
trazer distorções futuras em placas com muitos adultos. O rebalanceamento foi realizado principalmente com a obtenção de mais imagens de alados e ápteros. O número de ninfas foi mantido o mesmo do estudo anterior.

Dessa forma, foi retreinado a base do Rhopalosiphum padi com um dataset balanceado, resultando numa acurácia menor de validação em relação ao primeiro estudo, mesmo utilizando o mesmo modelo de treino: InceptionV3. A validação obtida foi de $86 \%$, longe de uma acurácia desejada, além de ser uma rede neural pesada para computadores convencionais ( $\approx 7$ milhões de parâmetros e modelo de $\approx 170 \mathrm{Mb}$ ).

Novamente, viu-se uma oportunidade de reduzir o tempo de processamento, motivo principal da reimplementação do software, juntamente com um aumento da acurácia utilizando uma rede neural convolucional própria. Ao ter liberdade de escolher os próprios parâmetros e tamanho de rede, criou-se um modelo mais preciso para o problema.

A rede neural criada consistiu, primeiramente, em trocar os canais de cores para preto e branco. Essa escolha se deu pois dentro da mesma espécie quase não há diferenciação de cores nas imagens, e utilizar três canais somente para classificar diferentes formatos poderia diminuir a acurácia. Dentro das camadas internas, utilizou-se a mesma técnica da Inception, com mais de uma convolução em cima da mesma camada, com uma concatenação de vetores na sequência.

Além disso, a nova rede se difere de algoritmos do estado da arte pelo seu tamanho reduzido, diminuindo vertiginosamente o tempo de classificação, além de exigir menor processamento. Os learning rates utilizados foram o mesmo do primeiro estudo, em 0.001, com 100 epochs de treinamento. Para a retropropagação foi utilizado o algoritmo de descida de gradiente estocástica, com um batch de 100 imagens. O treinamento se deu com data augmentation, utilizando rotação de 90 graus e giros verticais e horizontais das imagens.

4) Apresentação: O AphidCV 2.0 integrou os separados softwares CropAphid e AphidCV, exigindo agora o manejo de um único software por parte dos entomólogos para obter novos dados para treinamento de novas espécies e realizar a análise das placas. A nova interface (Figura 2) permite algumas funcionalidades novas, como, por exemplo, a visualização do efeito da variável do limiar em tempo real na placa escaneada, permitindo que os entomólogos escolham o valor que mais se adequa na detecção dos insetos.

Em relação às saídas após a análise, foram mantidas as mesmas da versão 1.0. Após rodar o software em cima de uma placa digitalizada, ele gera uma imagem processada de saída, com cada inseto circulado em cores diferentes, dependendo da classe determinada, como ilustra a Figura 3.

Além disso, o software gera planilhas eletrônicas com todas as informações do estágio de cada inseto detectado, juntamente com seu perímetro, altura, área e peso. O perímetro, a altura e a área são obtidos durante o encontro dos contornos, e o peso é calculado a partir de uma equação que relaciona peso com altura. Além das tabelas, o software gera um arquivo JSON, para possível uso em nuvem no futuro ou integração

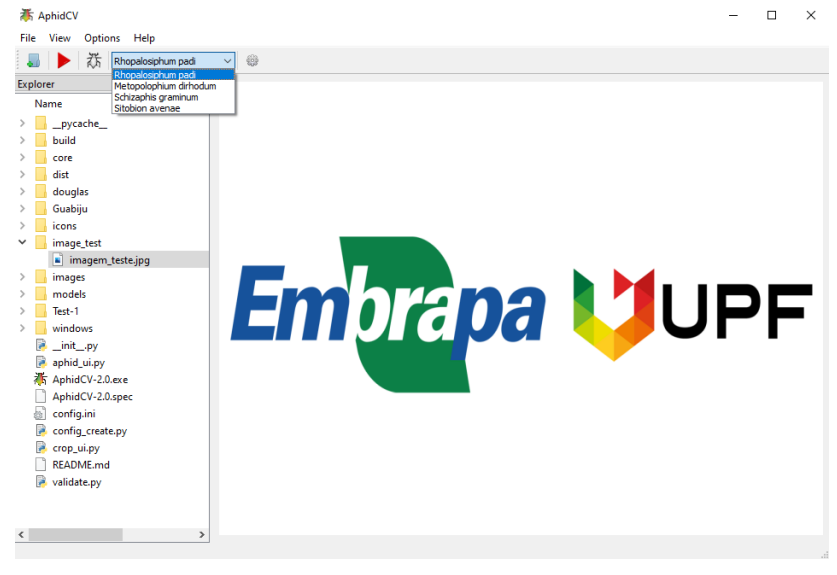

Figura 2. Interface gráfica do software AphidCV 2.0.

com outros softwares, como o de simulação de pragas que também está sendo desenvolvido na Embrapa Trigo.
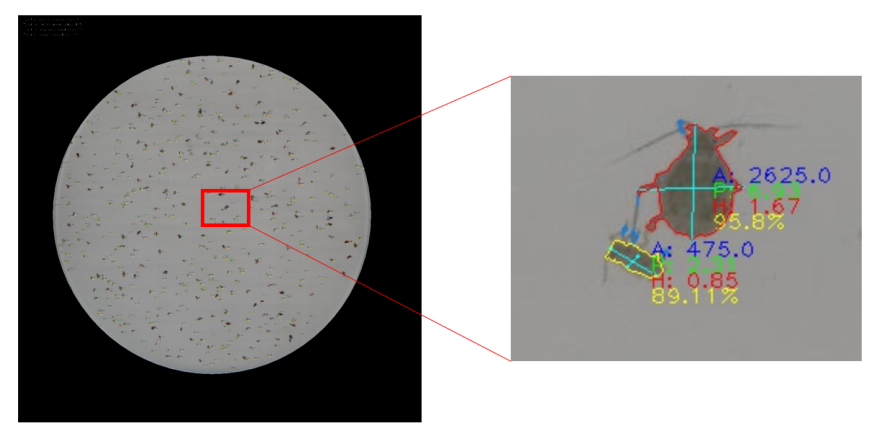

Figura 3. Exemplo de uma placa processada, com um zoom em um áptero e uma ninfa classificados.

\section{Resultados}

O software contemplou uma espécie nova e readequou o modelo inteligente para a espécie da primeira versão. $\mathrm{O}$ maior objetivo da reimplementação foi para diminuir o tempo de processamento e análise das placas. Os resultados obtidos são prévios, sendo necessário realizá-los de forma criteriosa em uma amostra grande de placas. Aqui, apresentamos os resultados de validação durante o treinamento.

A acurácia obtida durante a validação no treinamento foi de $97,8 \%$ para a espécie Rhopalosiphum padi e de $98,2 \%$ para a espécie Schizaphis graminum. A comparação com as acurácias da Inception e DenseNet-201, respectivamente, estão descritas na Tabela II. É necessário ressaltar que acurácia de validação não necessariamente resulta numa acurácia real similar. Isso se deve por possíveis sobreajustes após muitas rodadas de treinos, já que o dataset de validação também é levado em conta nos algoritmos de retropropagação durante o treino, mesmo que em menor proporção ao dataset de treinamento.

Com relação ao tempo de processamento, houve uma melhora exponencial. Para a análise, considerou-se quatro placas com cerca de 500 insetos cada. Houve uma redução de 10 vezes na velocidade de processamento $(90 \mathrm{~s})$ em relação à 
Tabela II

ACURÁCIA DE VALIDAÇÃo DE CADA UM DOS MODELOS TREINADOS.

\begin{tabular}{|c||c||c|}
\hline & R. padi & S. graminum \\
\hline InceptionV3 & $93,2 \%$ & $90,2 \%$ \\
\hline DenseNet-201 & $92,1 \%$ & $88,8 \%$ \\
\hline Rede neural própria & $97,8 \%$ & $98,2 \%$ \\
\hline
\end{tabular}

primeira versão do AphidCV (15 min), e 40 vezes mais rápida do que uma análise humana especializada (60 min).

\section{Discussão}

A segunda versão do AphidCV apresentou resultados melhores no desempenho, mantendo uma acurácia próxima da primeira versão. A hipótese de que a mudança da linguagem utilizada e o uso de um modelo de rede neural próprio para diminuir o tempo de processamento se mostrou verdadeira.

Entre os benefícios encontrados no uso da primeira versão, já se destacava a agilidade na análise de imagens das placas (quatro vezes mais rápida do que a análise manual), e uma eventual redução na fadiga do entomólogo, que poderia trabalhar na geração de resultados mais satisfatórios de contagem e de classificação, com melhor precisão de estimativas. Este fato manteve-se nessa nova versão.

Com um tempo de processamento reduzido em 40 vezes em relação ao manual na segunda versão, permite-se um aumento exponencial no volume de placas analisadas, permitindo a utilização de uma amostra muito maior dos campos analisados, adicionando precisão no controle dessas pragas.

Outro benefício do software, já contemplado na primeira versão, é a mensuração dos afídeos e o cálculo do peso dos mesmos, etapa comumente ignorada na contagem manual, por exigir muito tempo de análise. Nesse quesito, manteve-se as mesmas técnicas de processamento de imagens da primeira versão. Logo, espera-se que a acurácia no tamanho e peso dos afídeos continue similar com um tempo de processamento reduzido exponencialmente.

Em termos de acurácia, é necessário realizar testes com placas com maior diversificação no estágio de desenvolvimento e uma comparação com a primeira versão, já que a validação do primeiro estudo se deu em placas com muitas ninfas em um modelo possivelmente com sobreajuste. Porém, os números de validação pareceram promissores e próximos dos alcançados na primeira versão.

O AphidCV 2.0 já implementa a análise de mais duas novas espécies, necessitando apenas do modelo treinado de ambas, enquanto a primeira versão era específica de uma única espécie. No momento, a Embrapa Trigo está coletando e classificando as espécies Sitobion avenae e Metopolophium dirhodum, aumentando as espécies analisadas pelo software.

Dentre as limitações, está a não contemplação de afídeos sobrepostos, que acabam sendo recortados juntos durante o préprocessamento e são classificados como um só. Uma maneira computacional de resolver isso é utilizar técnicas de detecção de objetos. Entretanto, acredita-se que placas com um número menor de insetos mais espalhados resolvam bastante esses casos raros. Com o aumento exponencial no desempenho, análises de múltiplas placas se tornou mais viável.

\section{CONCLUSÃO}

O presente estudo apresentou uma nova versão do software AphidCV, um contador, classificador e mensurador de afídeos, utilizando técnicas de processamento de imagens, visão computacional e deep learning. Essa nova versão contemplou uma nova espécie, Schizaphis graminum, melhorou o modelo da espécie Rhopalosiphum padi, e diminuiu exponencialmente o tempo de análise das placas.

$\mathrm{O}$ uso de uma linguagem especializada em processamento massivo de imagens e redes neurais, juntamente com um modelo próprio de rede neural, muito mais leve do que o utilizado na primeira versão, permitiram com que o software realizasse uma análise em torno de 10 vezes mais rápida.

O método proposto é um importante passo para o uso de visão computacional na área de pesquisa em agricultura. A automação de contagem e classificação de afídeos auxilia na pesquisa de entomólogos, permitindo não só driblar problemas como a fadiga, como também aumentar o número de amostras, trazendo maior precisão na análise dessas pragas.

Trabalhos futuros incluem adicionar duas novas espécies, Sitobion avenae e Metopolophium dirhodum, e aumentar a acurácia dos atuais modelos treinados, com um aumento da base de treino. Além disso, pretende-se adicionar o software em nuvem, para que outros centros de pesquisas nacionais e internacionais possam usufruir do software.

\section{REFERÊNCIAS}

[1] R. Shavit, Z. S. Batyrshina, N. Dotan, and V. Tzin, "Cereal aphids differently affect benzoxazinoid levels in durum wheat," PloS one, vol. 13, no. 12 , p. e0208103, 2018.

[2] C.-A. Dedryver, A. Le Ralec, and F. Fabre, "The conflicting relationships between aphids and men: a review of aphid damage and control strategies," Comptes rendus biologies, vol. 333, no. 6-7, pp. 539-553, 2010.

[3] M. Yahya, N. A. Saeed, S. Nadeem, M. Hamed, and S. Shokat, "Role of wheat varieties and insecticide applications against aphids for better wheat crop harvest," Pakistan J. Zool, vol. 49, no. 6, pp. 2217-2225, 2017.

[4] A. Martin, D. Sathish, C. Balachander, T. Hariprasath, and G. Krishnamoorthi, "Identification and counting of pests using extended region grow algorithm," in Electronics and Communication Systems (ICECS), 2015 2nd International Conference on. IEEE, 2015, pp. 1229-1234.

[5] S. Shajahan, S. Sivarajan, M. Maharlooei, S. G. Bajwa, J. P. Harmon, J. F. Nowatzki, and I. Cannayen, "Identification and counting of soybean aphids from digital images using shape classification," Transactions of the Asabe, vol. 60, no. 5, pp. 1467-1477, 2017.

[6] N. Carter, A. F. G. Dixon, and R. Rabbinge, Cereal aphid populations: biology, simulation and prediction. Pudoc, 1982.

[7] J. G. A. Barbedo, "Using digital image processing for counting whiteflies on soybean leaves," Journal of Asia-Pacific Entomology, vol. 17, no. 4, pp. 685-694, 2014

[8] E. A. Lins, J. P. M. Rodriguez, S. I. Scoloski, J. Pivato, M. B Lima, J. M. C. Fernandes, P. R. V. da Silva Pereira, D. Lau, and R. Rieder, "A method for counting and classifying aphids using computer vision," Computers and Electronics in Agriculture, vol. 169, p. 105200 , 2020. [Online]. Available: http://www.sciencedirect.com/science/article/ pii/S0168169919306039

[9] P. Akulwar, "A recommended system for crop disease detection and yield prediction using machine learning approach," in Recommender System with Machine Learning and Artificial Intelligence: Practical Tools and Applications in Medical, Agricultural and Other Industries, S. N. M. and Jyotir Moy Chatterjee and Sarika Jain and Ahmed A. Elngar and Priya Gupta, Ed. John Wiley \& Sons, 2020, ch. 8, pp. 141-163. 